АНТИТЕЛА К СКЛЕРОСТИНУ КАК НОВАЯ АНАБОЛИЧЕСКАЯ ТЕРАПИЯ ОСТЕОПОРОЗА

( С. Е.О. Мамедова, Т.А. Гребенникова, Ж.Е. Белая, Л.Я. Рожинская

ФГБУ «Национальный медицинский центр эндокринологии» Минздрава России, Москва, Россия

Препараты для лечения остеопороза можно условно разделить на две группы: препараты, подавляющие костную резорбцию, и, соответственно, костеобразование (бисфосфонаты, деносумаб) и препараты, стимулирующие костеобразование, т.е. обладающие анаболическим действием. К последним относят терипаратид, паратгормон 1-84 и абалопаратид, которые, помимо стимуляции костеобразования, также стимулируют и костную резорбцию, что лимитирует их анаболический эффект.

Открытие склеростина - ключевого ингибитора костеобразования - привело к разработке концепций по ингибированию этого белка с целью усиления костеобразования. Ромосозумаб - человеческое моноклональное антитело к склеростину, которое, связываясь со склеростином, позволяет лигандам Wnt-сигнального пути взаимодействовать с их ко-рецепторами, что, в свою очередь, приводит к усилению костеобразования и повышению минеральной плотности кости. В отличие от классических анаболических препаратов для лечения остеопороза, ромосозумаб не только стимулирует костеобразование, но и подавляет костную резорбцию. В клинических исследованиях ромосозумаб продемонстрировал выраженный прирост минеральной плотности кости в позвоночнике и бедре. В статье представлены данные о доклинических и клинических исследованиях ромосозумаба.

КЛЮЧЕВЫЕ СЛОВА: Ромосозумаб; Склеростин; Антитела; Остеопороз

\title{
SCLEROSTIN ANTIBODIES AS NOVEL ANABOLIC THERAPY FOR OSTEOPOROSIS
}

\author{
(c) Elizaveta O. Mamedova, Tatiana A. Grebennikova, Zhanna E. Belaya, Liudmila Ya. Rozhinskaya
}

Endocrinology Research Centre, Moscow, Russia

Osteoporosis medications are divided into two groups: those inhibiting bone resorption and formation (bisphosphonates and denosumab), and those stimulating bone formation i.e. having an anabolic effect. The latter include teriparatide, parathyroid hormone 1-84 and abaloparatide, all of which stimulate bone resorption as well as bone formation, which limits their anabolic effect.

The discovery of sclerostin - the key inhibitor of bone formation - has led to development of the concept that inhibition of this protein could stimulate bone formation. Romosozumab is a human monoclonal antibody to sclerostin that binds to sclerostin and enables Wnt-signaling pathway ligands and their co-receptors to interact with each other, which, in turn, leads to increased bone formation and bone mineral density. Unlike classical anabolic drugs in osteoporosis treatment, romosozumab stimulates bone formation and inhibits bone resorption. In clinical trials, romosozumab showed marked increase in lumbar spine and hip bone mineral density. Presented article contains information about pre-clinical and clinical studies of romosozumab.

KEYWORDS: Romosozumab; Sclerostin; Antibodies; Osteoporosis

\section{ВВЕДЕНИЕ}

Открытие двух аутосомно-рецессивных заболеваний с высокой плотностью костной ткани, связанных с потерей экспрессии склеростина, вызвало интерес к роли этого белка в костном метаболизме. Склеростеоз и болезнь ван Бухема - два схожих заболевания, впервые описанные в 1950-х гг., развиваются вследствие мутаций, так или иначе затрагивающих ген SOST, приводя к потере кодируемого им белка - склеростина [1]. У пациентов со склеростеозом и болезнью ван Бухема наблюдается генерализованный склероз костей, что приводит к поВышению минеральной плотности костной ткани (МПК) во всех костях скелета. Однако клинически заболевания проявляют себя поражением костей черепа, приводящим к ограничению пространства для черепных нервов [2]. Склеростеоз в подавляющем большинстве случаев встречается среди африканеров Южной Африки, однако были описаны несколько случаев и в других странах. Пациенты обычно высокого роста, часто наблюдается синдактилия, которая может быть первым постнатальным признаком заболевания. Часто встречается компрессия черепных нервов, приводящая к параличам и глухоте. Утолщение свода черепа может приводить к угрожающему жизни повышению внутричерепного давления и требует проведения хирургической декомпрессии. При этом заболевании не отмечается повышения риска переломов, а отсутствие эктопической кальцификации и вовлечения других тканей указывает на то, что у пораженного гена отсутствуют множественные плейотропные эффекты [2]. В 2001 году две независимые группы исследователей показали, что причиной 
развития склеростеоза являются гомозиготные мутации в гене SOST, приводящие к образованию стоп-кодона и невозможности синтеза склеростина [3, 4]. Болезнь ван Бухема также является моногенным заболеванием с аутосомно-рецессивным типом наследования, характеризующимся генерализованным склерозом костей. Пациенты с этим заболеванием практически без исключения происходят из одной деревни в Нидерландах. Фенотип больных схож с таковым при склеростеозе, однако имеется ряд отличий: у пациентов обычно нормальный рост и отсутствуют синдактилии. В отличие от склеростеоза, при котором выявляются гомозиготные инактивирующие мутации в гене SOST, при болезни ван Бухема у пациентов выявляются гомозиготные делеции длиной 52 kb некодирующего энхансерного элемента, расположенного на 35 kb ниже гена SOST, необходимого для постнатальной экспрессии склеростина. У гетерозиготных носителей мутантных аллелей отмечается умеренное повышение костной плотности без других клинических проявлений [3].

Склеростин в основном экспрессируется в остеоцитах. Связывание с рецепторами LRP5 и LRP6 предотвращает активацию канонического Wnt-сигнального пути в кости, что снижает костеобразование [5]. Экспрессия склеростина может меняться в зависимости от ряда факторов, в частности глюкокортикоиды $[6,7]$ и гипергликемия повышают экспрессию склеростина, а паратгормон, физическая активность с осевыми нагрузками, половые гормоны снижают экспрессию склеростина [8]. Дей- ствие склеростина на Wnt-сигнальный путь реализуется не только в подавлении костеобразования, но, по некоторым данным также в повышении экспрессии рецептора активатора ядерного фактора каппа В (РАНКЛ) по сравнению с остеопротегерином, и, как следствие, усилении костного разрушения. Соответственно, при снижении уровня склеростина и активации Wnt-сигнала, происходит не только стимуляция остебластогенеза, но и изменение соотношения экспрессии РАНКЛ и остеопротегерина с преобладанием остеопротегерина и реализацией антирезорбтивного эффекта $[9,10]$. Основные эффекты действия склеростина в костной ткани суммированы на рисунке 1. С учетом механизма действия склеростина, в последующем были разработаны моноклональные антитела, которые связываются со склеростином и препятствуют его взаимодействию с LRP5/LRP6, что, в свою очередь, приводит к усилению костеобразования [1]. В доклинических и клинических исследованиях были исследованы несколько различных антител к склеростину: ромосозумаб (Amgen/UCB), блосозумаб (Eli Lilly) и BPS804 (Novartis). Наиболее исследованным из них является ромосозумаб $[11,12]$.

\section{РОМОСОЗУМАБ: ДОКЛИНИЧЕСКИЕ ИССЛЕДОВАНИЯ}

На стадии доклинических исследований было показано, что у грызунов с нокаутом гена склеростина (SOST) выявляется повышенная костная плотность, повышенное костеобразование как в кортикальном, так и тра-

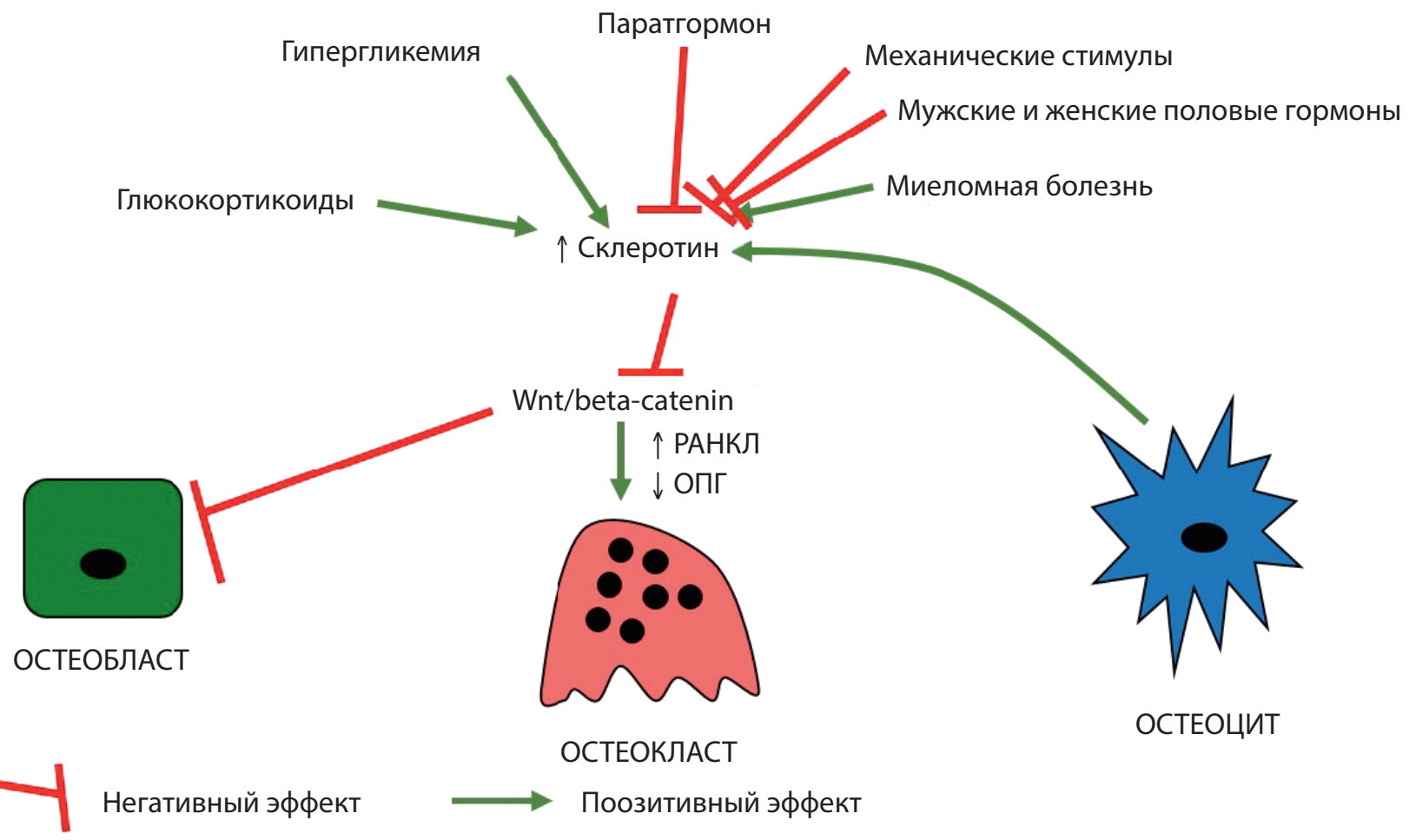

РАНКЛ - лиганд рецептора активатора ядерного фактора каппа В ОПГ - остеопротегерин

Рис. 1. Роль и механизмы регуляции склеростина в костной ткани.

Примечание. Экспрессия и содержание уровня склеростина регулируется гормональными и механическими стимулами. Повышение секреции уровня склеростина остеоцитами приводит к подавлению Wnt-сигнального пути и, как следствие, снижению остеобластогенеза. С другой стороны, из-за изменения соотношения РАНКЛ/ОПГ может активизироваться остеокластогенез и усиливаться костное разрушение. При связывании склеростина происходят прямо противоположные процессы, обусловленные активизацией Wnt-сигнального пути, усилением остеобластогенеза и костеобразование и снижением костной резорбции из-за изменения соотношения РАНКЛ и остеопротегерина с увеличением концентрации остеопротегерина 
бекулярном отделах кости, нормальное качество кости и повышенная прочность костей. Минерализация костного матрикса у животных с недостаточностью склеростина нормальная или снижена, что отражает отсутствие хрупкости костей, наблюдаемой, например, у пациентов с остеопетрозом вследствие дефицита или дисфункции остеокластов [13]. Исследования грызунов с нокаутом SOST подтвердили, что склеростин является отрицательным регулятором костной массы и костной прочности посредством ингибирования костеобразования in vivo [13]. Эти данные привели к появлению гипотезы, что фармакологическое подавление склеростина может иметь эффект у пациентов с низкой костной массой и высоким риском переломов, например, у пациентов с остеопорозом, посредством усиления костеобразования, увеличения костной массы и прочности костей.

Эффекты ромосозумаба на костеобразование, костную резорбцию, массу костной ткани, качество и прочность кости были исследованы на различных животных моделях с помощью исследования маркеров костного метаболизма, костной гистоморфометрии и изменений транскрипции в клетках кости [11]. Было установлено, что ромосозумаб способен активировать выстилающие клетки и стимулировать костеобразование на поверхности без предварительного этапа костной резорбции, то есть по типу моделирования костной ткани [14, 15]. При этом, усиление костеобразования отмечалось как на трабекулярных костных поверхностях в позвонках, так и эндокортикальных костных поверхностях в области проксимального отдела бедра $[16,17]$. Антитела к склеростину не оказывали значимого влияния на костеобразование в зонах костного ремоделирования, возможно за счет снижения количества поверхностей с костной резорбцией. Таким образом, антитела к склеростину усиливают костеобразование за счет увеличения продукции костного матрикса остеобластами и активации находящихся в состоянии покоя выстилающих клеток (так называемый процесс «моделирования» в противовес «ремоделированию» - классической схеме костеобразования) [11]. В животных моделях и у человека антитела к склеростину быстро повышают маркеры костеобразования, такие как $\mathrm{N}$-терминальный пропептид проколлагена 1 типа (P1NP), костно-специфическую щелочную фосфатазу и остеокальцин, при этом максимальный ответ достигается через 3 месяца от начала терапии, а через 6-12 месяцев уровень маркеров возвращается к исходному (так называемый феномен саморегулирования костеобразования) [14, 18]. Антитела к склеростину снижают костную резорбцию, однако неясно, посредством каких механизмов это происходит [11]. Двойной эффект антител к склеростину на повышение костеобразования и снижение костной резорбции приводит к повышению костной массы, улучшению микроархитектоники как трабекулярной, так и кортикальной кости. У овариэктомированных крыс наблюдалось повышение МПК по сравнению с контролем, как в позвоночнике, так и в бедре по данным рентгеновской денситометрии через 5 недель введения антител к склеростину [16, 19$]$.

Также на овариэктомированных крысах изучалось влияние предшествующей терапии остеопороза, одновременное назначение с антирезорбтивными препаратами и возобновление лечения антителами к скле- ростину после перерыва в лечении [11]. Так, переход на антитела к склеростину после предшествующего лечения алендроновой кислотой в течение 6 недель приводил к усилению костеобразования, дальнейшему приросту плотности и прочности кости [20]. Совместное назначение антител к склеростину с алендроновой кислотой не приводило к большему повышению костеобразования и костной массы по сравнению с контрольной группой [20], тогда как совместное назначение с золедроновой кислотой приводило к большему приросту костной массы по сравнению с группой контроля [21]. Назначение плацебо после лечения ромосозумабом у обезьян породы макак приводило к постепенному снижению костной массы [11]. В то же время, назначение алендроновой кислоты после ромосозумаба у овариэктомированных крыс или золедроновой кислоты у овариэктомированных мышей позволяло сохранить достигнутый на фоне лечения антителами к склеростину прирост МПК $[11,21]$. Таким образом, исследования на животных показали, что предшествующее лечение или совместное применение с бисфосфонатами не влияет на фармакологический эффект антител к склеростину. Эффект от лечения антителами к склеростину уменьшается при отмене терапии, может быть сохранен при последующем назначении антирезорбтивной терапии, восстанавливается после повторного назначения антител к склеростину после перерыва в лечении [11].

Эффекты антител к склеростину изучались также на животных моделях других видов остеопороза: мужского остеопороза, остеопороза вследствие иммобилизации, вследствие хронического воспаления, глюкокортикоидного остеопороза, остеопороза при поликистозе почек, сахарном диабете, множественной миеломе. На всех моделях отмечен положительный эффект антител к склеростину в предотвращении потери костной массы вследствие вышеперечисленных состояний [11].

\section{РОМОСОЗУМАБ: КЛИНИЧЕСКИЕ ИССЛЕДОВАНИЯ}

la фаза клинических исследований применения ромосозумаба у здоровых мужчин и женщин продемонстрировала уникальное двойное действие препарата на костный метаболизм, что соотносилось с результатами доклинических испытаний на животных моделях $[22,23]$. Ромосозумаб вводился как в режиме однократных, так и многократных инъекций: в дозе 1-2 мг/кг подкожно с частотой 1 раз в 2 недели или в дозе 2-3 мг/кг каждые 4 недели, в течение 3 месяцев. Изменение уровня маркеров костного ремоделирования отмечалось в первые 2 месяца терапии, при этом было максимальным в ответ на первую инъекцию препарата. Следует отметить, что фармакокинетика ромосозумаба не отличалась у мужчин и женщин. Изменения МПК были аналогичными при однократном и многократном режиме введения препарата. Так, через 85 дней после одной инъекции ромосозумаба отмечался прирост МПК в поясничных позвонках на 5,2\%, а в бедре (Total hip) на 1,1\% [22].

В рандомизированном двойном слепом плацебо-контролируемом клиническом исследовании Ib фазы оценивалось влияние ромосозумаба на плотность губчатого вещества и кортикального слоя посредством количе- 
ственной компьютерной томографии (ККТ) поясничных позвонков (L1-L2). В исследование было включено 48 пациентов (32 женщины и 16 мужчин) с низкой МПК, которые получали лечение препаратом в дозе 1-3 мг/кг каждые 2 недели в течение 3 месяцев и наблюдались на протяжении 3 месяцев после последней инъекции [24]. Спустя 3 месяца лечения ромосозумабом отмечалось значимое повышение минеральной плотности губчатого вещества и кортикального слоя на 9,5\% и 26,9\%, соответственно, по сравнению с группой плацебо (-3\% и -2,7\%, соответственно). Данные изменения МПК сохранялись в течение всего трехмесячного периода наблюдения [24].

Bo Ila плацебо-контролируемой фазе клинических исследований ромосозумаба оценивалась эффективность и безопасность различных доз препарата (70 мг, 140 мг и 210 мг), вводимых подкожно с интервалом в 1 или 3 месяца, у 419 женщин в постменопаузе с низкой МПК, чтобы определить оптимальный режим терапии [25]. В качестве групп сравнения были включены пациенты на терапии плацебо, алендроновой кислотой в дозе 70 мг/нед или терипаратидом 20 мкг/сут. Лечение ромосозумабом привело к быстрому и значительному увеличению маркеров образования костной ткани в сыворотке крови (P1NP и щелочной фосфатазы) при одновременном уменьшении маркера костной резорбции - С-концевого телопептида коллагена 1 типа (СТх). Интересно, что уровень P1NP достиг пика через 4 недели, вернулся к исходному между 3 и 6 месяцами терапии, после чего оставался ниже начального уровня на протяжении оставшегося периода лечения, в то время как СТх снижался после первой инъекции и оставался подавленным в течение всех 12 месяцев терапии. На фоне приема ромосозумаба во всех режимах дозирования отмечалось повышение МПК как в позвоночнике, так и в проксимальном отделе бедренной кости. Однако, наибольший прирост МПК через 12 месяцев лечения наблюдался при приеме ромосозумаба в дозе 210 мг 1 раз в месяц, в связи с чем именно данная схема дозирования препарата была выбрана для III фазы клинических исследований. За первый год лечения ромосозумабом МПК в поясничных позвонках и бедре увеличилась на 11,3\% и 4,1\%, соответственно, что превосходит эффект терипаратида и алендроновой кислоты. В течение же второго года терапии отмечалась меньшая прибавка МПК, что сочеталось со снижением уровня маркеров костеобразования. Таким образом, несмотря на то, что во время второго года лечения происходило дальнейшее увеличение МПК, наибольший эффект от терапии ромосозумабом был достигнут в течение первых 12 месяцев. [25].

B Ilb фазе клинических исследований лечение ромосозумабом продолжалось 2 года, однако была группа пациентов, которые в первый год получали ромосозумаб, а во второй год - плацебо. Как и следовало ожидать, в соответствии с клинической фармакологией ромосозумаба ингибирующее воздействие на маркеры ремоделирования кости резко прекратилось после завершения лечения. P1NP вернулся к уровню, наблюдаемому у пациентов, получавших плацебо, в то время как СТх увеличился выше уровня до лечения, прежде чем вернуться к исходному значению. МПК в позвоночнике и бедре возвратилась к уровню до лечения или приблизилась к ним. Подобные изменения наблюдались и при отмене блосозумаба после 12 месяцев лечения [25, 26]. Кроме того, схожее восстановление костного обмена и быстрое снижение МПК происходит после прекращения терапии деносумабом и оданакатибом [27, 28, 29, 30, 31]. Представленные результаты объясняют необходимость последующего лечения антирезорбтивным препаратом после отмены ромосозумаба для поддержания или возможного увеличения МПК. У пациентов, которые получили двухлетнее лечение ромосозумабом (в дозе 210 мг) и далее 1 год деносумабом, маркеры костного ремоделирования снизились и наблюдалось дальнейшее увеличение МПК. Так, суммарно за 3 года терапии остеопороза прибавка МПК составила 19,4\% в поясничном отделе позвоночника и 7,1\% в бедре [32]. Для сравнения: в течение двух лет монотерапии терипаратидом или в комбинации с деносумабом с последующим годом лечения деносумабом прирост МПК за 3 года в поясничных позвонках и бедре составил 15\% и 6\%, соответственно [33].

В четырех исследованиях III фазы оценивалось влияние ромосозумаба на снижение риска переломов. Первое из них, FRAME (Fracture Study in Postmenopausal Women with Osteoporosis) представляет собой международное рандомизированное двойное слепое плацебо-контролируемое исследование с участием 7180 женщин с постменопаузальным остеопорозом (средний возраст 71 год), которые получали ромосозумаб 210 мг ежемесячно или плацебо в течение первых 12 месяцев, а затем обе группы переходили на лечение деносумабом на протяжении также 12 месяцев [34]. Ромосозумаб снизил риск новых переломов позвонков на 73\% по сравнению с группой плацебо (0,5\% против 1,8\%, p<0,001). В группе пациенток, переключившейся с терапии ромосозумабом на деносумаб, риск переломов также был на 75\% ниже, чем в группе, перешедшей на него с плацебо (0,6\% против 2,5\%, p<0,001). Среди тех женщин, у которых рентгенография позвоночника была доступна для анализа, переломы тел позвонков в первые 12 месяцев исследования зафиксированы у 16 из 3321 пациенток в группе ромосозумаба и у 59 из 3322 пациенток в группе плацебо. Следует отметить, что только 2 из 16 переломов в группе ромосозумаба случились после полугода лечения, все остальные - в первые 6 месяцев. Интересно, что была выявлена зависимость между снижением риска переломов позвонков и географическим расположением исследовательского центра. Оказалось, что в странах Латинской Америки риск переломов позвонков был намного ниже, чем в других частях мира, что влияло на результаты исследования, исключая действие ромосозумаба на этот параметр [34]. Однако после исключения данного региона из статистического анализа данных эффект ромосозумаба на снижение риска переломов позвонков был статистически подтвержден. Таким образом, по результатам исследования FRAME терапия ромосозумабом снижает частоту новых случаев переломов тел позвонков (на 73\%) и частоту клинических симптомов переломов (на 36\%), однако снижение частоты внепозвоночных переломов данное исследование не выявило [34].

В другом исследовании III фазы - ARCH (Active-Controlled Fracture Study in Postmenopausal Women with Osteoporosis at High Risk), производилась оценка влияния ромосозумаба на снижение риска переломов у женщин с постменопаузальным остеопорозом и наличием пере- 
ломов в анамнезе. Всего в исследование было включено 4093 пациентки, которые в течение первого года получали ромосозумаб 210 мг/мес или алендроновую кислоту 70 мг/нед, после чего переводились на терапию алендроновой кислотой в течение дополнительных двух лет [35]. В отличие от плацебо-контролируемого исследования FRAME в ARCH были включены пациентки с более высоким риском переломов, соответствовавших следующим критериям включения: (1) Т-критерий МПК -2,5 или ниже в бедре (Total hip или Neck) в сочетании с одним или более переломами позвонков (в зависимости от степени компрессии); или (2) Т-критерий МПК -2 или ниже в бедре (Total hip или Neck) в сочетании с двумя и более переломами тел позвонков или переломом проксимального отдела бедренной кости, которые произошли за 3-24 месяца до рандомизации. Средний возраст участников исследования - 74,3 года, при этом более половины женщин были в возрасте 75 лет и старше. Средние значения T-критерия L1-L4 и Total hip составили -2,96 и -2,8, соответственно. [35]. После 12 месяцев терапии новые переломы позвонков произошли у 4\% пациенток в группе ромосозумаба и у 6,1\% женщин, получавших алендроновую кислоту, что привело к относительному снижению риска переломов позвонков по сравнению с алендроновой кислотой на 37\% ( $p=0,003)$. Риск клинических переломов позвонков был снижен на $28 \%(p<0,001)$ в группе ромосозумаба по сравнению с алендроновой кислотой. Риск внепозвоночных переломов был ниже на 26\% при использовании ромосозумаба через 12 месяцев, однако результат не был статистически значимым $(p=0,06)$. МПК поясничных позвонков увеличилась на 13,7\% после 12 месяцев лечения ромосозумабом по сравнению с 5\% в группе терапии алендроновой кислотой. Аналогично, увеличение МПК проксимального отдела бедренной кости было больше в группе ромосозумаба $(6,2 \%)$ по сравнению с алендроновой кислотой (2,8\%). [35].

Преимущество лечения ромосозумабом по влиянию на снижение риска компрессионных переломов тел позвонков сохранялась и в течение второго года исследования после перехода на терапию алендроновой кислотой. По сравнению с группой, получавшей алендроновую кислоту 2 года, риск переломов позвонков был на 48\% ниже (6,2\% против 11,9\%) у женщин, принимавших 1 год ромосозумаб и 1 год алендроновую кислоту. Во время второго года исследования на фоне приема алендроновой кислоты новые компрессионные переломы развились у 45 пациенток, которые ранее принимали ромосозумаб, против 115 пациенток, принимавших алендроновую кислоту оба года. МПК у женщин, получавших ромосозумаб, а затем алендроновую кислоту, за 2 года лечения увеличилась суммарно на 15,2\% и 7,1\% в L1-L4 и Total hip, соответственно. Кроме того, в исследовании ARCH было доказано, что ромосозумаб в течение 12 месяцев с последующим приемом алендроновой кислоты снижал риск новых переломов позвонков в течение 24 месяцев на 48\%, внепозвоночных - на 19\% и переломов бедра на 38\% по сравнению с монотерапией алендроновой кислотой. Однако, в группе ромосозумаба серьезные нежелательные явления со стороны сердечно-сосудистой системы наблюдались чаще, чем в группе терапии алендроновой кислотой (50 из 2040 пациентов (2,5\%) против 38 из 2014 пациентов (1,9\%), соответственно) [35]. Вместе с тем, в плацебо-контролируемом исследовании FRAME подобной разницы не было обнаружено.

В другом рандомизированном открытом клиническом исследовании III фазы- STRUCTURE, действие ромосозумаба сравнивалось с терипаратидом у 436 женщин с постменопаузальным остеопорозом, которые ранее уже получали терапию бисфосфонатами в течение не менее 3-х лет (средняя продолжительность лечения 5,6 лет) [36]. В исследование включались пациентки с Т-критерием МПК -2,5 и ниже в бедре (Total hip или Neck) или поясничных позвонках и переломом В анамнезе. В течение года пациентки получали или ромосозумаб 210 мг/мес, или терипаратид 20 мкг/сут. В группе ромосозумаба после года терапии прирост МПК в бедре составил 2,6\%, в то время как в группе терипаратида отмечалось снижение на 0,6\%. По результатам ККТ бедра повышение минеральной плотности трабекулярной костной ткани наблюдалось в обеих группах, однако кортикальная кость увеличилась только в группе ромосозумаба $(+1,1 \%)$, тогда как на фоне лечения терипаратидом отмечалась отрицательная динамика (-3,6\%). Кроме того, следует отметить, что частота серьезных нежелательных явлений была сопоставима в обеих группах терапии [36].

Эффективность и безопасность ромосозумаба в лечении остеопороза у мужчин оценивалась в рандомизированном плацебо-контролируемом исследовании III фазы - BRIDGE, в которое было включено 245 мужчин (55 лет и старше) с остеопорозом и низкотравматичными переломами (кроме переломов бедра) или компрессионными переломами тел позвонков в анамнезе [37]. Пациенты были рандомизированы в отношении 2:1 в группу лечения ромосозумабом 210 мг/мес (163 пациента) и группу плацебо (82 пациента). После года лечения ромосозумабом прирост МПК по сравнению с плацебо в поясничных позвонках составил 12,1\% против 1,2\%, в бедре в целом (Total hip) 2,5\% против -0,5\% и в шейке бедра 2,2\% против -0,2\% ( $<<0,001)$. Однако, как и в исследовании ARCH, были выявлены серьезные нежелательные явления со стороны сердечно-сосудистой системы в группе пациентов, получавших лечение ромосозумабом (4,9\% против 2,5\% в группе плацебо) [37]. Результаты клинических исследований суммированы в таблице 1.

\section{БЕЗОПАСНОСТЬ РОМОСОЗУМАБА}

В целом, по данным клинических исследований, ромосозумаб хорошо переносился. Незначительные реакции в месте введения наблюдались у 4,4\%-5,2\% исследуемых, получавших 210 мг ромосозумаба один раз в месяц (по сравнению с 2,6\%-2,9\% в группе контроля) [34, 35]. У одного пациента в I фазе клинического исследования отмечалось транзиторное симптоматическое повышение уровня трансаминаз, однако в целом нарушения функции печени при применении ромосозумаба не наблюдались [23]. Нечасто встречалось транзиторное и бессимптомное снижение уровня кальция в крови с реципрокным увеличение показателя ПТГ во II фазе исследования, обычно при более высоких дозах препарата [25]. Антитела к ромосозумабу были обнаружены у 15-20\% пациентов в течение первого года 
Таблица 1. Общие данные об эффективности ромосозумаба в клинических исследованиях

Общая характеристика ис-
следования (название; доза и
кратность введения ромосо-
зумаба и группы сравнения;
продолжительность исследо-
вания; ссылка)

\section{la фаза;}

доза 1-2 мг/кг подкожно с

частотой 1 раз в 2 недели или в дозе 2-3 мг/кг каждые 4 недели; продолжительность - 3 месяца; [21].

\section{Ib фаза;}

доза 1-3 мг/кг каждые 2 недели; продолжительность 6 месяцев

(3 месяца на терапии и 3 месяца наблюдения);

[23].

\section{Эффектив- \\ ность для \\ предупреж- \\ дения пере- \\ ломов тел \\ позвонков \\ Эффек- \\ тивность \\ для преду- \\ преждения \\ внепозво- \\ ночных \\ переломов}

Эффективность для
прибавки MПК (по-
ясничные позвон-
ки; бедро VS группа
сравнения)

48 пациентов (32 женщины в постменопаузе и 16 мужчин) с МПК от -2,5SD до -1SD.

L1-L4 +5,2\%, в бедре

(Total hip) $+1,1 \%$.

Возраст: 45-80 лет.

48 пациентов (32 женщины в постменопаузе и 16 мужчин) с МПК от -2,5SD до -1SD.

Возраст: 45-80 лет.
Прирост МПК губчатого вещества (L1-L2) на $+9,5 \%$ vs $-3 \%$ плацебо и кортикального слоя (L1-L2) +26,9\% vs $-2,7 \%$.

L1-L4 +11,3\% (vs плацебо -0,1\%; алендроновая кислота $+4,1 \%$;

II фаза; дозы 70 мг, 140 мг и 210 мг с интервалом в 1 или 3 месяца; продолжительность - 12 месяцев; группы сравнения - алендроновая кислота в дозе 70 мг/ нед и терипаратид 20мкг/сут; [24].
419 женщин в постме-

нопаузе с низкой МПК

(менее -2,5SD);

возраст - 55-85 лет терипаратид $+7,1 \%)$, все бедро $+4,1 \%$

(vs плацебо -0,7\%; алендроновая кислота $+1,9 \%$; терипаратид $+1,3 \%)$, шейка бедра

$+3,7 \%$ (vs плацебо $-1,1 \%$; алендроновая кислота $+1,2 \%$; терипаратид $+1,1 \%)$.

III фаза FRAME;

В течение 12 месяцев: доза ромосозумаба 210 мг/мес, группа сравнения - плацебо; следующие 12 месяцев обе группы деносумаб 60 мг/6 месяцев; [33].

III фаза ARCH;

12 месяцев - ромосозумаб 210 мг/мес или алендроновая кислота 70 мг/нед, далее 24 месяца - алендроновая кислота 70 мг/ нед;

[34].
7180 женщин с постменопаузальным остеопорозом (средний возраст 71 год)
На $73 \%$ по сравнению с группой плацебо
Не выявлено статистически значимой разницы
L1-L4 13,3\% vs плацебо, все бедро $+6,9 \%$, шейка бедра $+5,9 \%$.
4093 пациентки в постменопаузе: (1) Т-критерий МПК -2,5 или ниже в бедре в сочетании с одним или более переломами тел позвонков (в зависимости от степени компрессии); или (2) Т-критерий МПК -2 или ниже в бедре в сочетании с двумя и более переломами позвонков или переломом проксимального отдела бедренной кости, которые произошли за 3-24 месяца до рандомизации. Средний возраст

\footnotetext{
Через 12 ме- Через 12 месяцев - на $37 \%$ сяцев - на $26 \%$ (по сравнению (по сравнению алендроновая кислота с алендроно- с салендроно- +5\%) и бедро +6,2\% вой кислотой), вой кислотой), (vs алендроновая кисчерез 24 меся- через 24 меся- лота $+2,8 \%$ ) ца - на $48 \%$ ца - на $38 \%$
} 


\begin{tabular}{|c|c|c|c|c|}
\hline $\begin{array}{c}\text { Общая характеристика ис- } \\
\text { следования (название; доза и } \\
\text { кратность введения ромосо- } \\
\text { зумаба и группы сравнения; } \\
\text { продолжительность исследо- } \\
\text { вания; ссылка) }\end{array}$ & $\begin{array}{c}\text { Количество пациен- } \\
\text { тов; описание иссле- } \\
\text { дуемой популяции } \\
\text { (пол, средний возраст, } \\
\text { тяжесть остеопороза) }\end{array}$ & $\begin{array}{l}\text { Эффектив- } \\
\text { ность для } \\
\text { предупреж- } \\
\text { дения пере- } \\
\text { ломов тел } \\
\text { позвонков }\end{array}$ & $\begin{array}{c}\text { Эффек- } \\
\text { тивность } \\
\text { для преду- } \\
\text { преждения } \\
\text { внепозво- } \\
\text { ночных } \\
\text { переломов }\end{array}$ & $\begin{array}{c}\text { Эффективность для } \\
\text { прибавки МПК (по- } \\
\text { ясничные позвон- } \\
\text { ки; бедро VS группа } \\
\text { сравнения) }\end{array}$ \\
\hline
\end{tabular}

III фаза STRUCTURE;

ромосозумаб 210 мг/мес или терипаратид 20 мкг/сут; продолжительность - 12 месяцев; [35].
436 женщин с постменопаузальным остеопорозом: Т-критерием МПК -2,5 и ниже в бедре (Total hip или Neck) или поясничных позвонках и переломом в анамнезе. Ранее

пациентки получали терапию бисфосфонатами на менее 3-х лет.
245 мужчин (163 в группу

ромосозумаба и 82 в группу плацебо) с остеопорозом и низкотравматичными переломами (кроме переломов бедра) или компрессионными переломами тел позвонков в анамнезе. Возраст:
L1-L4 +9,8\% vs +5,4\% (терипаратид), Total hip $+2,6 \%$ vs $-0,6 \%$ ), Neck $+3,2 \%$ vs $-0,2 \%$.
III фаза BRIDGE; ромосозумаб 210 мг/мес, группа сравнения - плацебо; [36].

55 лет и старше.

терапии, из них у 3\% антитела имели нейтрализующую активность in vitro, однако данных о том, что они влияли на эффективность и безопасность препарата получено не было [34, 35]. В исследовании FRAME было два $(<0,1 \%)$ случая остеонекроза челюсти и один $(<0,1 \%)$ случай атипичного перелома бедра [34].

Учитывая, что пролиферация многих клеток в организме находится под контролем канонического Wht-сигнального пути, существовало опасение о возможности развития злокачественных новообразований на фоне терапией ингибиторами склеростина. В исследовании ромосозумаба на крысах данных о развитии злокачественных новообразований получено не было [37]. В исследовании FRAME не было выявлено повышения частоты сердечно-сосудистых событий на фоне приема ромосозумаба (сердечно-сосудистые события встречались в 1,1\% женщин, получающих плацебо, и в 1,2\% среди получавших ромосозумаб) [34]. Смертность составила 0,8\% в группе ромосозумаба и 0,6\% - в группе плацебо [34]. Однако в исследовании ARCH через 12 месяцев терапии в группе ромосозумаба отмечалось значимое повышение частоты сердечно-сосудистых событий и инсульта $(0,8 \%)$ по сравнению с группой алендроновой кислоты (0,3\%) [35]. Тридцать из 2040 женщин (1,5\%), получающих ромосозумаб, умерли в течение первого года исследования $\mathrm{ARCH}$, по сравнению с 21 из 2014 в группе, получающих алендроновую кислоту (1\%). Разница в 0,5\% в смертности сохранялась в течение всего периода наблюдения, когда уже все участницы получали алендроновую кислоту [35]. Механизм побочного действия ромосозумаба на сердечно-сосудистую систему остается неясен, также как и причины различия в частоте сердечно-сосудистых событий меж- ду плацебо-контролируемым исследованием FRAME и сравнительным исследованием ARCH. Необходимо также отметить, что мРНК склеростина экспрессируется и в других органах и тканях, в частности в сердце, аортальных клапанах, головном мозге, почках. Однако, как правило, обнаружив мРНК ученым не удавалось найти белок склеростина в этих органах [8]. Одним из объяснений феномена расхождения данных исследований FRAME и ARCH по безопасности ромосозумаба может служить положительный эффект бисфосфонатов на сердечно-сосудистый риск и летальность у пациентов с тяжелым остеопорозом. Так, в рамках многоцентрового рандомизированного плацебо-контролируемого исследования эффективности золедроновой кислоты у пациентов с переломом бедра было продемонстрировано снижение смертности у пациентов, получивших золедроновую кислоту в течение трех лет, по сравнению с плацебо [38]. Причем этот эффект было нельзя объяснить снижением риска переломов. Данные наблюдательных исследований и мета-анализов также демонстрируют снижение риска летальности и сердечно-сосудистых осложнений на фоне терапии бисфосфонатами [39], хотя некоторые исследования указывают на нейтральный эффект [40]. Возможно, положительный эффект бисфосфонатов на летальность и сердечно-сосудистые заболевания и осложнения удается зарегистрировать только в популяциях с тяжелым остеопорозом и высоким риском всех неблагоприятных исходов. В исследование, сравнивающее ромосозумаб и алендроновую кислоту, как раз и были включены пациенты с тяжелым остеопорозом, что могло отразить благоприятный эффект бисфосфонатов, а не неблагоприятный эффект ромосозумаба. 


\section{ЗАКЛЮЧЕНИЕ}

Ромосозумаб представляет новый класс антиостеопоротической терапии. На этапе доклинических и клинических исследований был продемонстрирован уникальный двойной эффект ромосозумаба на костную ткань и доказана эффективность терапии: быстрое повышение МПК в течение первого года лечения, снижение риска как позвоночных, так и внепозвоночных переломов.
Отсутствие последействия препарата на костную ткань компенсируется последующим назначением бисфосфонатов для поддержания достигнутого эффекта.

\section{ДОПОЛНИТЕЛЬНАЯ ИНФОРМАЦИЯ}

Конфликт интересов. Авторы декларируют отсутствие явных и потенциальных конфликтов интересов, связанных с публикацией настоящей статьи.

\section{СПИСОК ЛИТЕРАТУРЫ | REFERENCES}

1. Holdsworth G, Roberts SJ, Ke HZ. Novel actions of sclerostin on bone. J. Mol. Endocrinol. 2019:R167-R185. doi: https://doi.org/10.1530/jme-18-0176

2. van Lierop AH, Appelman-Dijkstra NM, Papapoulos SE. Sclerostin deficiency in humans. Bone. 2017;96:51-62. doi: https://doi.org/10.1016/j.bone.2016.10.010

3. Balemans W. Identification of a $52 \mathrm{~kb}$ deletion downstream of the SOST gene in patients with van Buchem disease. J. Med. Genet. 2002;39(2):91-97. doi: https://doi.org/10.1136/jmg.39.2.91

4. Brunkow ME, Gardner JC, Van Ness J, et al. Bone Dysplasia Sclerosteosis Results from Loss of the SOST Gene Product, a Novel Cystine Knot-Containing Protein. The American Journal of Human Genetics. 2001;68(3):577-589. doi: https://doi.org/10.1086/318811

5. Гребенникова Т.А., Белая Ж.Е., Солодовников А.Г. и др. Wnt10b n Wnt3a как биомаркеры изменений регуляции костного обмена у пациентов с болезнью Иценко-Кушинга // Вестник Российской академии медицинских наук. - 2018. - Т. 73. - № 2. - С. 115-121. [Grebennikova TA, Belaya ZE, Solodovnikov AG et al. Wnt10b and Wnt3a as biomarkers of changes in the regulation of bone metabolism in patients with Cushing's Disease. Vestnik Rossijskoj akademii medicinskih nauk. 2018;73(2):115-121. (In Russ.)] doi: https://doi. org/10.15690/vramn904

6. Belaya ZE, Grebennikova TA, Melnichenko GA et al. Effects of endogenous hypercortisolism on bone mRNA and microRNA expression in humans. Osteoporosis International. 2018;29(1): 211-221. https://doi.org/10.1007/s00198-017-4241-7

7. Belaya ZE, Rozhinskaya LY, Melnichenko GA et al. Serum extracellular secreted antagonists of the canonical Wnt/ $\beta$-catenin signaling pathway in patients with Cushing's syndrome. Osteoporosis International. 2013;24:2191-2199. https://doi.org/10.1007/s00198-013-2268-y

8. Delgado-Calle J, Sato AY, Bellido T. Role and mechanism of action of sclerostin in bone. Bone. 2017:96:29-37.

9. Гребенникова Т.А., Белая Ж.Е., Рожинская Л.Я. и др. Эпигенетические аспекты остеопороза. Вестник Российской академии медицинских наук. - 2015. - Т. 70. - №5. - С. 541-548. [Grebennikova TA, Belaya ZE, Rozhinskaya LYa et al. Epigenetic aspects of osteoporosis. Vestnik Rossijskoj akademii medicinskih nauk. 2015;70(5):541-548. (In Russ.)] doi: https://doi.org/10.15690/vramn.v70.i5.1440

10. Гребенникова Т.А., Белая Ж.Е., Рожинская Л.Я., Мельниченко Г.А. Канонический сигнальный путь Wnt/ß-катенин: от истории открытия до клинического применения. Терапевтический архив. - 2016. - Т. 88. - №10. - С. 74-81. [Grebennikova TA, Belaya ZE, Rozhinskaya LYa, Melnichenko GA. The canonical $\mathrm{Wnt} / \beta$-catenin pathway: from the history of its discovery to clinical application. Terapevticheskij arhiv. 2016;88(10):74-81. (In Russ.)] doi: https://doi.org/10.17116/terarkh201688674-81

11. Ominsky MS, Boyce RW, Li X, Ke HZ. Effects of sclerostin antibodies in animal models of osteoporosis. Bone. 2017;96:63-75. doi: https://doi.org/10.1016/j.bone.2016.10.019

12. McClung MR. Romosozumab for the treatment of osteoporosis. Osteoporosis and Sarcopenia. 2018:4(1):11-15. doi: https://doi.org/10.1016/j.afos.2018.03.002

13. Li X, Ominsky MS, Niu Q-T, et al. Targeted Deletion of the Sclerostin Gene in Mice Results in Increased Bone Formation and Bone Strength. J. Bone Miner. Res. 2008;23(6):860-869. doi: https://doi.org/10.1359/jbmr.080216

14. Nioi P, Taylor S, Hu R, et al. Transcriptional Profiling of Laser Capture Microdissected Subpopulations of the Osteoblast Lineage Provides Insight Into the Early Response to Sclerostin Antibody in Rats. J. Bone Miner. Res. 2015;30(8):1457-1467. doi: https://doi.org/10.1002/jbmr.2482
15. Kim SW, Lu Y, Williams EA, et al. Sclerostin Antibody Administration Converts Bone Lining Cells Into Active Osteoblasts. J. Bone Miner. Res. 2017;32(5):892-901. doi: https://doi.org/10.1002/jbmr.3038

16. Ominsky MS, Niu Q-T, Li C, et al. Tissue-Level Mechanisms Responsible for the Increase in Bone Formation and Bone Volume by Sclerostin Antibody. J. Bone Miner. Res. 2014;29(6):1424-1430. doi: https://doi.org/10.1002/jbmr.2152

17. Ominsky MS, Vlasseros F, Jolette J, et al. Two doses of sclerostin antibody in cynomolgus monkeys increases bone formation, bone mineral density, and bone strength. J. Bone Miner. Res. 2010;25(5):948-959. doi: https://doi.org/10.1002/jbmr.14

18. Ominsky MS, Samadfam R, Jolette J et al. Long-term sclerostin antibody treatment in cynomolgus monkeys: sustained in vertebral microarchitecture and bone strength following a temporal increase in cancellous bone formation [abstract]. J Bone Miner Res. 2012;27 (Suppl 1). doi: https://doi.org/10.1002/jbmr.1852

19. Li X, Ominsky MS, Warmington KS, et al. Sclerostin Antibody Treatment Increases Bone Formation, Bone Mass, and Bone Strength in a Rat Model of Postmenopausal Osteoporosis. J. Bone Miner. Res. 2009;24(4):578-588. doi: https://doi.org/10.1359/jbmr.081206

20. Li X, Ominsky MS, Warmington KS, et al. Increased Bone Formation and Bone Mass Induced by Sclerostin Antibody Is Not Affected by Pretreatment or Cotreatment with Alendronate in Osteopenic, Ovariectomized Rats. Endocrinology. 2011;152(9):3312-3322. doi: https://doi.org/10.1210/en.2011-0252

21. Halleux C, Hu S, Diefenbach B et al. Infrequent co-treatment and sequential treatment of anti-sclerostin antibody with zoledronic acid restores and maintains bone mass in murine osteoporosis models. J Bone Miner Res. 2009;24 (Suppl 1). doi: https://doi.org/10.1002/jbmr.5650241301

22. Padhi D, Allison M, Kivitz AJ, et al. Multiple doses of sclerostin antibody romosozumab in healthy men and postmenopausal women with low bone mass: A randomized, double-blind, placebo-controlled study. The Journal of Clinical Pharmacology. 2014;54(2):168-178. doi: https://doi.org/10.1002/jcph.239

23. Padhi D, Jang G, Stouch B, et al. Single-dose, placebo-controlled, randomized study of AMG 785, a sclerostin monoclonal antibody. J. Bone Miner. Res. 2011;26(1):19-26. doi: https://doi.org/10.1002/jbmr.173

24. Graeff C, Campbell GM, Peña J, et al. Administration of romosozumab improves vertebral trabecular and cortical bone as assessed with quantitative computed tomography and finite element analysis. Bone. 2015;81:364-369. doi: https://doi.org/10.1016/j.bone.2015.07.036

25. McClung MR, Grauer A, Boonen S, et al. Romosozumab in Postmenopausal Women with Low Bone Mineral Density. N. Engl. J. Med. 2014;370(5):412-420. doi: https://doi.org/10.1056/NEJMoa1305224

26. Recknor CP, Recker RR, Benson CT, et al. The Effect of Discontinuing Treatment With Blosozumab: Follow-up Results of a Phase 2 Randomized Clinical Trial in Postmenopausal Women With Low Bone Mineral Density. J. Bone Miner. Res. 2015;30(9):1717-1725. doi: https://doi.org/10.1002/jbmr.2489

27. Bone $\mathrm{HG}$, Bolognese MA, Yuen CK, et al. Effects of Denosumab Treatment and Discontinuation on Bone Mineral Density and Bone Turnover Markers in Postmenopausal Women with Low Bone Mass. J. Clin. Endocr. Metab. 2011;96(4):972-980. doi: https://doi.org/10.1210/jc.2010-1502

28. Cummings SR, Ferrari S, Eastell R, et al. Vertebral Fractures After Discontinuation of Denosumab: A Post Hoc Analysis of the Randomized Placebo-Controlled FREEDOM Trial and Its Extension. J. Bone Miner. Res. 2018;33(2):190-198. doi: https://doi.org/10.1002/jbmr.3337 
29. Heiss G. Health Risks and Benefits 3 Years After Stopping Randomized Treatment With Estrogen and Progestin. JAMA. 2008;299(9):1036. doi: https://doi.org/10.1001/jama.299.9.1036

30. Wasnich RD, Bagger YZ, Hosking DJ et al. Changes in bone density and turnover after alendronate or estrogen withdrawal. Menopause. 2004; $11(6$ Pt 1):622-630.

31. Binkley N, Krueger D, de Papp AE. Multiple vertebral fractures following osteoporosis treatment discontinuation: a case-report after long-term Odanacatib. Osteoporos. Int. 2018;29(4):999-1002. doi: https://doi.org/10.1007/s00198-018-4385-0

32. Papapoulos S, Lippuner K, Roux C, et al. The effect of 8 or 5 years of denosumab treatment in postmenopausal women with osteoporosis: results from the FREEDOM Extension study. Osteoporos. Int. 2015;26(12):2773-2783. doi: https://doi.org/10.1007/s00198-015-3234-7

33. Leder BZ, Tsai JN, Uihlein AV, et al. Denosumab and teriparatide transitions in postmenopausal osteoporosis (the DATA-Switch study): extension of a randomised controlled trial. The Lancet. 2015;386(9999):11471155. doi: https://doi.org/10.1016/s0140-6736(15)61120-5

34. Cosman F, Crittenden DB, Adachi JD, et al. Romosozumab Treatment in Postmenopausal Women with Osteoporosis. N. Engl. J. Med. 2016;375(16):1532-1543. https://doi.org/10.1056/NEJMoa1607948

35. Saag KG, Petersen J, Brandi ML, et al. Romosozumab or Alendronate for Fracture Prevention in Women with Osteoporosis. N. Engl. J. Med. 2017;377(15):1417-1427. doi: https://doi.org/10.1056/NEJMoa1708322
36. Langdahl BL, Libanati C, Crittenden DB, et al. Romosozumab (sclerostin monoclonal antibody) versus teriparatide in postmenopausal women with osteoporosis transitioning from oral bisphosphonate therapy: a randomised, open-label, phase 3 trial. The Lancet. 2017;390(10102):1585-1594. doi: https://doi.org/10.1016/s0140-6736(17)31613-6

37. Lewiecki EM, Blicharski T, Goemaere S, et al. A Phase III Randomized Placebo-Controlled Trial to Evaluate Efficacy and Safety of Romosozumab in Men With Osteoporosis. J. Clin. Endocr. Metab. 2018;103(9):3183-3193. doi: https://doi.org/10.1210/jc.2017-02163

38. Chouinard L, Felx M, Mellal N, et al. Carcinogenicity risk assessment of romosozumab: A review of scientific weight-of-evidence and findings in a rat lifetime pharmacology study. Regul. Toxicol. Pharm 2016;81:212-222. doi: https://doi.org/10.1016/j.yrtph.2016.08.010

39. Lyles KW, Colón-Emeric CS, Magaziner JS et al. HORIZON Recurrent Fracture Trial. Zoledronic acid and clinical fractures and mortality after hip fracture. N Engl J Med. 2007;357(18):1799-809. https://doi.org/10.1056/NEJMoa074941

40. Kranenburg G, Bartstra JW, Weijmans M et al. Bisphosphonates for cardiovascular risk reduction: A systematic review and meta-analysis. Atherosclerosis. 2016;252:106-115. https://doi.org/10.1016/j.atherosclerosis.2016.06.039

41. Kim DH, Rogers JR, Fulchino LA et al. Bisphosphonates and risk of cardiovascular events: a meta-analysis. PLoS One. 2015;10(4):e0122646. https://doi.org/10.1371/journal.pone.0122646

\section{ИНФОРМАЦИЯ ОБ АВТОРАХ [AUTHORS INFO]}

* Мамедова Елизавета Октаевна, к.м.н. [Elizaveta O. Mamedova, MD, PhD]; адрес: (почтовый рабочий адрес с индексом) Россия, 117036 Москва, ул. Дмитрия Ульянова, д.11 [address: 11 Dm.Ulyanova street, 117036 Moscow, Russia]; ORCID: http://orcid.org/0000-0002-9783-3599; eLibrary SPIN: 3904-6017; e-mail: lilybet@mail.ru

Гребенникова Татьяна Алексеевна, К.М.н. [Tatiana A. Grebennikova, MD, PhD]; e-mail: grebennikova@hotmail.com; ORCID: http://orcid.org/0000-0003-1413-1549; eLibrary SPIN: 4380-5447.

Белая Жанна Евгеньевна, д.М.н. [Zhanna E. Belaya, MD, PhD]; e-mail: jannabelaya@gmail.com; ORCID: http://orcid.org/0000-0002-6674-6441; eLibrary SPIN: 4746-7173.

Рожинская Людмила Яковлевна, д.М.Н., профессор [Liudmila Ya. Rozhinskaya, MD, PhD, Professor]; e-mail: Irozhinskaya@gmail.com; ORCID: http://orcid.org/0000-0001-7041-0732; eLibrary SPIN: 5691-7775.

\section{ЦИТИРОВАТЬ:}

Мамедова Е.О., Гребенникова Т.А., Белая Ж.Е., Рожинская Л.Я. Антитела к склеростину как новая анаболическая терапия остеопороза. // Остеопороз и остеопатии. - 2018. - Т. 21. — №3. - C.21-29. doi: https://doi.org/10.14341/osteo10127

\section{TO CITE THIS ARTICLE:}

Mamedova EO, Grebennikova TA, Belaya ZhE, Rozhinskaya LYa. Sclerostin antibodies as novel anabolic therapy for osteoporosis. Osteoporosis and bone diseases. 2018;21(3):21-29. doi: https://doi.org/10.14341/osteo10127 